\title{
Managing Children With Renal Diseases During the COVID-19 Pandemic
}

\author{
Anil Vasudevan ${ }^{1}$, Mukta Mantan ${ }^{2}$, Sriram Krishnamurthy ${ }^{3}$, Priya Pais ${ }^{1}$, Georgie Mathew ${ }^{4}$,

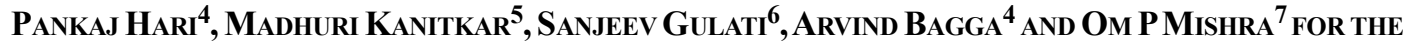 \\ Indian Society of Pediatric Nephrology \\ ${ }^{1}$ Department of Pediatric Nephrology, St. John's Medical College Hospital, Bengaluru, Karnataka; ${ }^{2}$ Department of Pediatrics, \\ Maulana Azad Medical College, New Delhi; ${ }^{3}$ Department of Pediatrics, JIPMER, Puducherry; ${ }^{4}$ Division of Pediatric Nephrology, All \\ India Institute of Medical Sciences, New Delhi; ${ }^{5}$ DCIDS Medical, Jammu and Kashmir; ${ }^{6}$ Department of Nephrology, Fortis Group of \\ Hospitals, New Delhi, ${ }^{7}$ Division of Pediatric Nephrology, Department of Pediatrics, Institute of Medical Sciences, Banaras Hindu \\ University, Varanasi, Uttar Pradesh; India. \\ Correspondence to: Dr Om P Mishra, Professor, Division of Pediatric Nephrology, Department of Pediatrics, Institute of Medical \\ Sciences, Banaras Hindu University, Varanasi 221 005, Uttar Pradesh, India.opmpedia@yahoo.co.uk
}

The coronavirus outbreak is a rapidly evolving pandemic, placing unprecedented strain on health-care systems. COVID-19 presents challenges for management of children with renal diseases, especially those receiving long-term immunosuppressive medications, including renal transplant recipients and those with chronic kidney disease and acute kidney injury requiring dialysis. Our preparedness for managing this vulnerable group of children is the need of the hour. The purpose of this article is to provide guidance to caregivers and health care personnel involved in management of children with renal diseases and to ensure patient well-being, while protecting staff from infection.

Keywords: Chronic kidney disease, Hemodialysis, Nephrotic syndrome, Transplant.

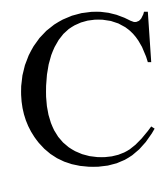
oronavirus disease 2019 (COVID-19) has been declared as a pandemic, given its global spread. Children account for $1-5 \%$ patients and are less likely to become severely ill compared to adults; though, preschool children and infants might have severe clinical features [1,2].

In March, 2020, the Indian Society of Pediatric Nephrology (ISPN) decided to formulate guidelines on managing children with renal diseases during the COVID19 pandemic. A writing committee and advisory board was formed to draft guidelines, based on policies and guidelines from Ministry of Health and Family Welfare, Indian Society of Nephrology and international professional organizations, and evidence from systematic and narrative reviews, trials and other reports. Draft guidelines underwent multiple iterations before being finalized.

\section{Are Patients With Kidney Disease At Risk for COVID-19 and Poor Outcomes?}

Co-morbidities associated with mortality during COVID19 are common in adult patients with chronic kidney disease (CKD), and those on maintenance dialysis. Children with CKD especially stage $4-5$, those on hemodialysis (HD) or receiving immunosuppressive agents are considered immunocompromised. Patients with advanced CKD are malnourished and undergo maintenance HD in busy units, increasing the risk of infection. Analysis of confirmed patients with COVID-19 reported to the Center for Disease Control (USA) revealed that patients with CKD were 11 to 14-times more likely to be hospitalized and require intensive care, respectively compared to those without CKD [3].

Reports from China suggest a less severe course of the disease in dialysis compared to transplant recipients. At a dialysis center in Renmin Hospital, Wuhan, 37 of 230 patients on HD and 4 of 33 dialysis staff showed severe acute respiratory syndrome coronavirus-2 (SARS-CoV-2) infection over 4-weeks [4]. Seven patients died, of which 6 had COVID-19. The incidence of COVID-19 in HD patients reported from China is similar to that from a similar cohort in Italy. Of 20 adult transplant recipients in Brescia (Lombardy, Italy) with COVID-19, 5 died, 4 were critically ill and 3 recovered. Similarly, among $21 \mathrm{HD}$ patients with COVID-19; 5 died while 4 recovered [5]. In a report on 15 adult transplant recipients with COVID-19 from a single center in US, one-fourth were ventilated with one death, while $50 \%$ were discharged [6]. In contrast, another report from US reported a higher mortality among 36 adult kidney-transplant recipients with Covid-19 compared to general population as well as to patients more than 70 years old with COVOD-19 (28\% vs 5\% and 8$15 \%$, respectively) [7]. However, none of the transplanted or dialyzed children were infected in Lombardy that 
reported more than 8000 patients with COVID-19 [personal communication: G. Montini, Pediatric Nephrology Unit, Milano].

Experts feel that there is evidence that adult patients with CKD, especially those on dialysis, transplant recipients or receiving immunosuppressive therapy, are at increased risk for SARS-CoV-2 infection, with significant morbidity and unsatisfactory outcomes.

\section{Recommendations for Patients and their Caregivers}

Caregivers refer to parents/guardians taking care of health and personnel needs of children. Patients with $\mathrm{CKD}$, those on immunosuppressive medications and transplant recipients, and their caregivers should follow appropriate advice to reduce the risk of getting sick. These measures include self-isolating and staying at home to minimize contact between people; avoiding nonessential travel, crowded places and large gatherings; washing hands frequently with soap and water; and adopting cough etiquette. Patients and caregivers should wear a triple-layer mask while visiting healthcare facility including dialysis units. In outpatient clinic, social distancing measures should be strictly followed along with other measure of personal protection. All used disposable gloves and masks should be placed in a lined container before disposing them with other household waste and wash hands with soap and water/alcoholbased hand rub.

Caregivers should ensure around 4 weeks stock of medications at home. They should contact their treating physician or hospital, by phone or email, if child has fever, cough, shortness of breath, with or without rhinorrhea, and muscle aches or chills.

\section{Healthcare Personnel}

Health care personnel (HCP) refer to those directly related to provision of health care services. HCP should receive information about COVID-19, and training on institutional and national protocols for evaluation and management [8]. Doctors, dialysis nurses and technicians must follow guidelines for prevention and control of infections and adhere to protocols for identifying and reporting patients of COVID-19. Clinical management of patients with COVID19 is evolving, and doctors are advised to stay updated.

Teleconsultations with patients and their families are encouraged to minimize hospital visits. Simple strategies are employed to support mental well-being of children and their families, and mitigate anxiety and stress [9]. All staff members in the dialysis unit should be trained in donning and doffing of PPE [10].
Doctors, dialysis nurses and technicians should stagger their schedule to reduce exposure to infection, and have a reserve force that could be deployed for management of patients [11]. We endorse recommendations of the National Taskforce for hydroxychloroquine (HCQ) prophylaxis, for HCP involved in care of suspected or confirmed patients with COVID-19, and household contacts of laboratory confirmed cases [12]. However, there is a need to be cautious and avoid its indiscriminate use due to potential cardiac and other toxicities. This practice may change as more evidence emerges on benefits and safety of its use. HCQ should not be used for prophylaxis in children younger than 15 year and those with glucose-6phosphate dehydrogenase deficiency. Caregivers and the patient should be informed about the rationale of therapy, contraindications and adverse effects.

\section{CHILDREN RECEIVING IMMUNOSUPPRESSIVE THERAPY}

Immunosuppression and anti-proteinuric measures are cornerstones of treatment in renal diseases. Immunosuppression was one of the most common underlying conditions in a report on 345 children with COVID-19 [2]. Patients receiving therapy with the following agents should be considered immuno-compromised: Corticosteroids (prednisolone, methylpre-dnisolone, dexamethasone): Prednisolone dose $>20 \mathrm{mg}$ daily for $>4-$ weeks in the last 6-months, or $>5 \mathrm{mg}$ daily for $>4$ weeks with one or more immunosuppressive agents in last 6-months; Calcineurin inhibitors (tacrolimus, cyclosporine); Mycophenolate mofetil, azathioprine; Cyclophosphamide: any dose (oral or intravenous) within the last 6 months; Rituximab: any dose within the last 6 months; and, Plasma exchange in the preceding 6-weeks [13].

\section{General Management}

We advise that dose of immunosuppressive medication should not be changed, since the risk of disease flare is higher than the threat posed by COVID-19 in children. Patients should be advised to keep $~ 4$-weeks stock of immunosuppressive medications. Hospital visits for nonemergency purposes are avoided and HCP contacted through telecommunication. The physician may consider deferring maintenance doses of IV cyclophosphamide or rituximab in patients in sustained remission, and low risk of relapse on case to case basis. Patients should be encouraged to maintain hydration.

\section{Nephrotic Syndrome, Glomerulonephritis, Vasculitis}

We recommend that the first episode and relapse of nephrotic syndrome should be treated promptly with 
standard dose of prednisolone, as under normal circumstances. Delayed initiation of therapy might result in complications associated with anasarca and bacterial infections. Continuation of therapy may be discussed telephonically. No changes should be made in ongoing treatment of frequent relapsing and steroid resistant nephrotic syndrome.

Decisions regarding initiating immunosuppressive therapy in newly diagnosed patients with other glomerular diseases or vasculitis, especially those from hotspots/ clusters should be based on disease severity, renal histology and serum creatinine, severity of proteinuria and co-morbidities, and balancing the risk versus benefit of therapy [14]. We advise initiating immunosuppression in newly diagnosed patients with glomerular diseases or vasculitis, according to existing guidelines, except in children with asymptomatic or low-grade proteinuria and normal renal function. These patients may be initially managed with salt restriction, and blood pressure control using an angiotensin converting enzyme inhibitor or angiotensin receptor blocker (ACE-I/ARB). As with nephrotic syndrome, no changes are advised in ongoing or proposed immunosuppressive therapy for patients with other glomerular disorders or vasculitis.

\section{Angiotensin Converting Enzyme Inhibitors (ACE-I) and Angiotensin Receptor Blockers (ARB)}

SARS-CoV-2, binds to its receptor, ACE-2 for entry into target cells suggesting an increased ability of the virus to enter the lungs in patients on ACE-I or ARB [15]. While there is debate regarding safety of inhibitors of reninangiotensin-aldosterone axis, few studies have evaluated their effects on severity of illness or mortality in COVID19. Thus, therapy with ACE-I or ARBs should be continued [16]. Abrupt discontinuation of medications may be associated with uncontrolled hypertension, and its consequences.

\section{Hydroxychloroquine (HCQ)}

Therapy with HCQ should continue in patients who are already receiving the medication, e.g., for systemic lupus, vasculitis. Risk of depleting stock of HCQ needs to be considered and adequate stocks of medication should be ensured.

\section{Renal Biopsy}

It is suggested to prioritize patients with indications for renal biopsy during the pandemic. Biopsies that are necessary and have therapeutic implications, e.g., suspected allograft rejection, rapidly progressive glomerulonephritis, small vessel vasculitis and interstitial nephritis should be performed while those for less emergent indications, e.g., steroid resistant nephrotic syndrome, calcineurin toxicity and persistent hematuria may be delayed.

\section{Patients on Immunosuppression With Suspected or Confirmed COVID-19}

Children on immunosuppression may present with mild symptoms but have high risk of deterioration and require hospitalization. Early identification of severe pneumonia and severe acute respiratory illness (SARI) is important as it allows prompt admission to a designated hospital ward or intensive care unit, and initiation of treatment. Patient with suspected COVID-19 should be shifted to an isolation facility or designated COVID area as soon as possible.

An approach to management of a child on immunosuppressive medications with respiratory symptoms is summarized in Fig. 1. While there is no specific guidance on precise modification of immunosuppression, it seems prudent to reduce or withhold immunosuppressive medications, except stress doses of steroids in patients with severe COVID-19 requiring admission to intensive care units.

Given the lack of specific treatment, most patients with COVID-19 require supportive care alone. More than 500 trials evaluating 150 drugs are being conducted worldwide [17]. Consistent with national guidelines, we suggest use of HCQ (7-8 mg/kg/dose twice daily for day 1, and 7-8 mg/ $\mathrm{kg}$ once a day from 2-6 days for $>12-\mathrm{yr}$ ) in patients with severe disease and requiring ICU care [18]. The doses should be reduced by $50 \%$ for children in CKD stage 5 and those on dialysis. Caregivers should be informed about the rationale of therapy, and potential adverse effects, especially prolonged QTc interval. There is insufficient data to recommend the use of remdesivir, lopinavir/ ritonavir or other HIV protease inhibitors for patients.

\section{CHILDREN WITH CHRONIC KIDNEY DISEASE}

Since children with CKD stage 3-5 are considered vulnerable to infection with coronavirus, it is vital that children and caregivers follow above mentioned precautions. Patients and caregivers should maintain contact with their physicians, especially for symptoms of COVID-19 including fever and worsening respiratory symptoms. Paracetamol is safe for children with fever, but treatment with other non-steroidal anti-inflammatory drugs (ibuprofen, naproxen) should be avoided.

Patients should continue taking antihypertensive medications, targeting systolic and diastolic blood pressures to $\sim 90^{\text {th }}$ percentile for age, gender and height. We recommend continued therapy with ACE-I or ARB in patients with CKD who are receiving such therapy. 
Fever, cough, and/or shortness of breath

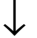

Wear mask

Transfer to triage are of hospital

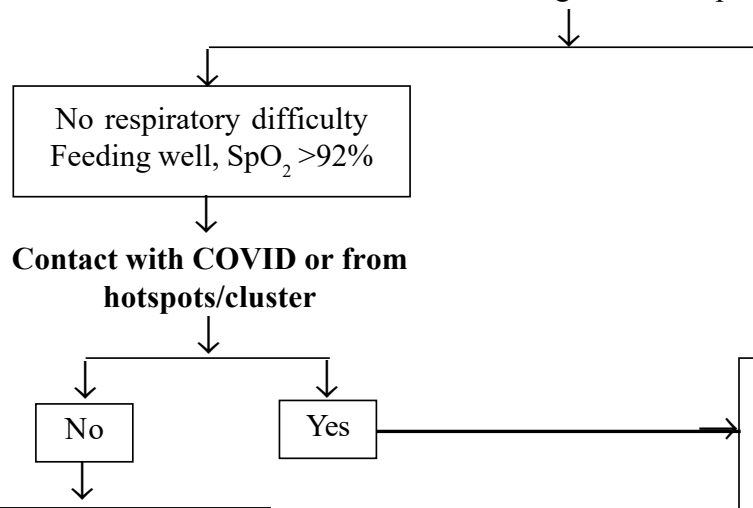

Respiratory difficulty Not Feeding well, $\mathrm{SpO}_{2}<92 \%$
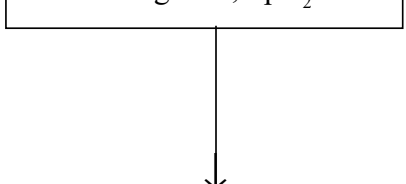

Transfer to COVID ward/unit RT-PCR; Chest $X$-ray, lab tests Initiate supportive care

Home management Empirical antibiotics Wear mask; hand hygiene; cough etiquette Explain danger signs; worsening symptoms
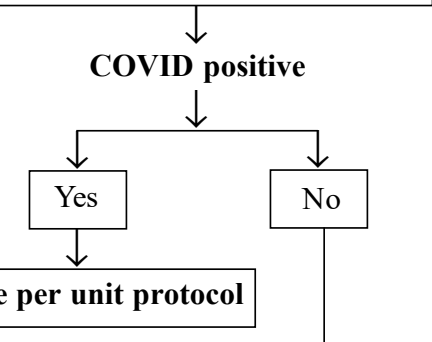

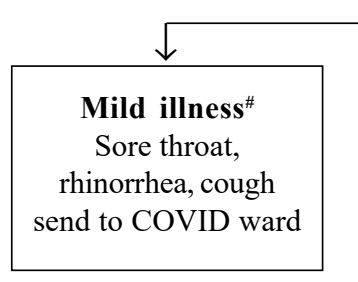

Oral antibiotics Monitor closely Adequate fluids Paracetamol Steroids (stress)

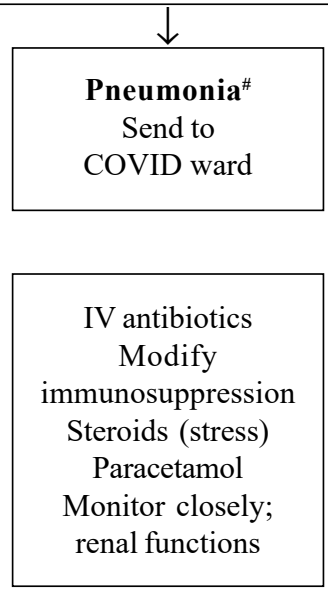

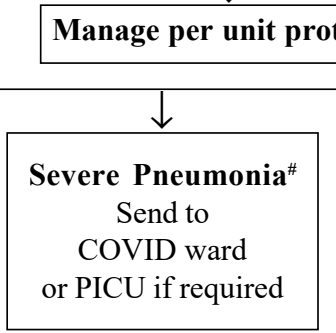

IV antibiotics

$\mathrm{O}_{2}$ (prongs, HFNC);

Avoid nebulizer; use

MDI, spacer Antiviral therapy Modify immunosuppression steroids (stress) Paracetamol Monitor closely; renal functions

Fig.1 Triaging and management of child on immunosuppression or with chronic kidney disease. (HFNC-high flow nasal cannula, MDI: metered dose inhaler, PICU: pediatric intensive care unit, RRT: renal replacement therapy) *Testing: 'Nasopharyngeal, posteriorpharyngeal swab; endotracheal aspirate or BAL:RT PCR for COVID-19. Evaluate with blood counts (CBC); renal and liver function tests; coagulogram, Ddimer, fibrinogen; C-reactive protein; chest radiograph; blood culture ${ }^{\#}$ Non-severe Pneumonia: Cough or difficulty in breathing with fast breathing (<2-months: $=60 ; 2-11$ months: $=50 ; 1-5$ years: $=40$ breaths per minute); and no signs of severe pneumonia. "Severe pneumonia: Cough or difficulty in breathing, with at least one of the following: (i) central cyanosis or oxygen saturation (SpO2) <90\%; (ii) severe respiratory distress (grunting, very severe chest indrawing); (iii) signs of pneumonia with one danger sign: inability to breastfeed/drink, lethargy/unconsciousness, or convulsions. Signs of pneumonia: chest indrawing, fast

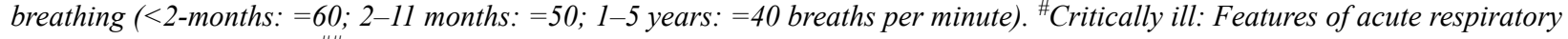
distress syndrome; shock ${ }^{\#} H C Q$ - Hydroxychloroquine 7-8 mg/kg/dose BD for day 1; 7-8 mg/kg once a day from days 2-6; avoid concomitant azithromycin since it can lead to arrhythmias. (Doses for children are extrapolated from that recommended in adults). 
Abrupt withdrawal of these agents might result in clinical instability and adverse outcomes.

Children on chronic ambulatory peritoneal dialysis (CAPD) should continue sessions at home following the standard protocol and precautions, avoid hospital visits, and maintain adequate stock of fluids and consumables. Automated PD (APD) machine should be disinfected using 70\% alcohol-based solution before and after each treatment. They should keep in contact with the doctor or dialysis nurse, and inform promptly for fever, symptoms ofCOVID-19 and peritonitis.

If COVID-19 is suspected in CKD, then patient should be shifted to an isolation facility if available or to designated COVID hospital as soon as possible and managed as per standard guidelines (Fig. 1).

\section{Hemodialysis (HD) Units}

Inpatient and outpatient pediatric dialysis facilities must be prepared for patients infected with SARS-CoV-2. The Ministry of Health and Family Welfare has prepared comprehensive guidelines for HD of COVID-19 patients [19]. The statement below is adapted from the above guidance, specifically addressing needs for children.

Children require $\mathrm{HD}$ in two situations: (i) maintenance HD for end stage renal disease, (ii) dialysis for acute kidney injury (AKI), related or unrelated to COVID-19.

An outbreak of COVID 19 in a dialysis facility is defined as two or more COVID-19 infections resulting from a common exposure, that is either suspected or laboratory-confirmed as SARS-CoV-2 [20]. After identification, the outbreak should be reported to the hospital authorities and appropriate measures should be taken as per Government of India guidelines [8].

\section{General Recommendations}

- Patients on HD are advised not to postpone their dialysis schedule. Phone numbers and contact information of the dialysis unit should be provided to the patients.

- Administrators need to ensure availability of consumables, including dialysate, dialyzers and tubing, catheters, fistula needles, disinfectants and medications.

- It is necessary to educate $\mathrm{HCP}$, patients and caregivers about COVID-19, including hand hygiene, respiratory hygiene and cough etiquette, use of facemasks and disposal of contaminated items. Posters and literature (in local language) should be available.

- All dialysis personnel should use appropriate PPE, as per institutional policy.

- Dialysis waiting area, beds and nursing station(s) should be equipped with alcohol-based sanitizers along with paper napkins and foot operated plastic lined waste disposal bins.

- Duties of HCP should be organized as per institutional policy, with an overall aim to maintain a pool of reserve staff.

- Bed side rounds by group of staff, group-studies and office case-discussions involving teams should be minimized.

- Patients with features suggestive of respiratory infections (fever, cough) should be identified before they enter the dialysis area. Caregivers are instructed to call the unit to report fever or respiratory symptoms, so that they are directed to an appropriate triage in the hospital. At each dialysis visit, a staff member must perform a structured interview for patient and caregivers, asking for: history of fever, cough, respiratory difficulty and exposure to a patient with COVID-19.

- Children should be advised to use a triple-layer facemask while in the waiting area, during dialysis and until they reach home. Seats in the waiting area should be separated by at least 1 meter. To avoid overcrowding, children should be accompanied by only one attendant who should also wear a facemask.

- Dialysis patients, who have a parent or family member on 14-days quarantine, should continue to receive HD during this period. Once the family members or caregiver are confirmed SARS-CoV-2 positive, the dialysis patient should be isolated and instructed to call the unit before arriving for subsequent HD sessions, and to report fever or respiratory symptoms.

\section{Dialysis Unit: During Dialysis and Disinfection}

- If feasible, dialysis beds should be spaced at a minimum distance of 2 meters.

- Patients are instructed to wash their hands and fistula arm before starting dialysis. Puncture sites should be cleaned, and appropriately disinfected.

- Disposable gloves should be used when handling laundry from infected patients. Dirty laundry should not be shaken to minimize the possibility of dispersing virus through air.

- Bed linen should be changed between shifts, and used linen placed in dedicated containers.

- Disposable gowns must be discarded after use. Cloth 
gowns are soaked in $1 \%$ hypochlorite solution for 20 minutes before sluicing, and transported to laundry [19].

- All surfaces and equipment in the unit should be cleaned and disinfected at least once daily, and after each patient shift. This includes bedside tables and lockers, dialysis machines, patient monitors, syringe pumps, sphygmomanometers, doorknobs, lightswitches, counter tops, handles, desks, phones, keyboards, toilets, faucets and sinks. For surfaces such as carpeted floor, rugs and drapes, visible contamination is removed, followed by appropriate cleaners indicated for these surfaces. After cleaning, items should be laundered in accordance with manufacturer instructions and dried completely.

- Disinfection is done with either $1 \%$ bleach solution or $70 \%$ alcohol-based solution. Bleach is preferred for surfaces that do not soak up water (example: floor). Use of $70 \%$ alcohol based solution is recommended for disinfection of metallic surfaces like door knobs or handles

\section{Dialyzing Patients With Suspected or Confirmed COVID-19}

Most pediatric hemodialysis units in developing countries are small, comprising 3- 6 beds. In order to prevent transmission of infection, it is advised that patients with suspected or confirmed COVID-19 be dialyzed in a separate room, with separate access and with the door closed. If a separate room is not available, the suspected patient may be dialyzed in a corner or end-ofrow station, maintaining at least 2-meters separation in all directions, preferably in the last shift of the day. The patient, as well as other patients should wear 3 layered masks while dialysis personnel, should wear appropriate PPE throughout the procedure.

Units that do not have enough space and/or dedicated work force for dialyzing suspected or confirmed COVID-19 patients should facilitate their transfer to a designated adult or pediatric HD units until the testing is negative. This plan should be communicated to the caregivers, which will help them prepare accordingly.

- It is recommended to use separate equipment, including stethoscopes, thermometers, saturation probes, and blood pressure cuffs, with cleaning and disinfection between shifts. Stethoscopes are disinfected with alcohol-based solutions. Dialysis personnel should not touch the patient or use stethoscopes, unless necessary.
- Surfaces and equipments located within 1-meter of the patient should be disinfected, as detailed previously. All disposable supplies are discarded.

- Dialysis personnel taking care of a patient with suspected COVID-19 should not look after other patients during the same shift. Staff should self-report symptoms of fever, cough or breathlessness.

- Institutional and national guidelines should be followed for managing patients with suspected or confirmed COVID (Fig. 2).

\section{Personal Protective Equipment (PPE) for Dialysis Personnel}

- Dialysis personnel shall be instructed regarding the need for personal protection. They will be trained for donning and doffing of PPE, its proper use and disposal [10].

- We suggest the use of triple-layer masks, head cover, gloves, water-impermeable gown and shoe-covers for HCP working in the unit. Personnel involved in procedures involving aerosol generation, venepuncture and dialysis access should follow standard contact and droplet precautions, and should wear N95 mask and disposable face-shield [21]. Table I summarizes the protective equipment required for different levels of anticipated contact.

- Further, in children requiring plasmapheresis using HD machine and in the HD unit, all measures suggested in the hemodialysis section should be followed.

\section{Dialyzing Children With COVID-19 and Acute Kidney Injury}

The incidence of AKI in patients with COVID-19 ranges from $6-15 \%$ [22]. Patients with CKD on maintenance dialysis may require care in an intensive care unit. A proportion of patients with secondary bacterial infection will have septic shock, drug nephrotoxicity or worsening of existing CKD, severe enough to require renal replacement therapy (RRT).

The goal is to deliver RRT in a safe and timely manner. Children may need to be dialyzed in shared spaces with adults, if dedicated space is not available. Centres should anticipate surge in COVID-19 related AKI and the need for dialysis may outpace available facilities. As in any patient with AKI, indications for initiating RRT and choice of modality i.e., peritoneal dialysis, $\mathrm{HD}$, continuous renal replacement therapy (CRRT), and sustained low efficiency dialysis (SLED), is based on resources and expertise, and patient hemodynamic status. We suggest the following: 

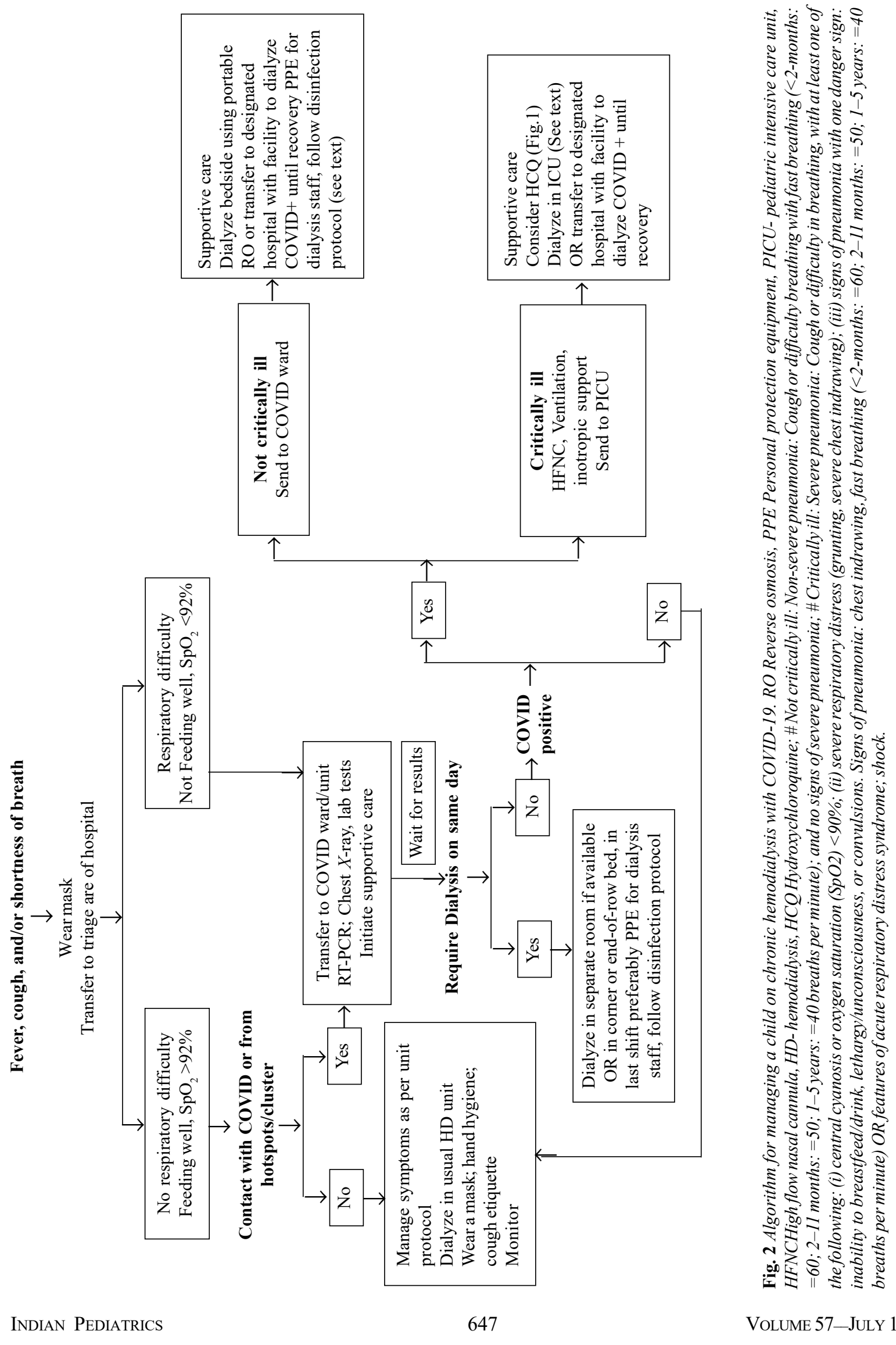

Volume 57_JuLy 15, 2020 
Table I Protective Equipment Required for Different Levels of Anticipated Contact

\begin{tabular}{|c|c|c|c|c|c|c|c|}
\hline Category of staff & $\begin{array}{l}\text { Hand } \\
\text { hygiene }\end{array}$ & $\begin{array}{l}\text { Eye } \\
\text { protection* }\end{array}$ & N95 mask & $\begin{array}{l}\text { Surgical } \\
\text { mask }\end{array}$ & Apron & Gowns & Gloves \\
\hline Reception staff & Yes & - & - & Yes & - & Yes & Yes \\
\hline Triage staff & Yes & - & - & Yes & - & Yes & Yes \\
\hline $\begin{array}{l}\mathrm{HCP} \text { attending to the patient } \\
\text { (routine examination) }\end{array}$ & Yes & Yes & - & Yes & Yes & - & Yes \\
\hline $\begin{array}{l}\text { HCP performing procedures like } \\
\text { HD/PD in non-COVID case }\end{array}$ & Yes & Yes & - & $\begin{array}{l}\text { Yes/N95 } \\
\text { extended use }\end{array}$ & - & Yes & Yes \\
\hline $\begin{array}{l}\mathrm{HCP} \text { performing aerosol } \\
\text { generating procedures or } \mathrm{HD} / \mathrm{PD} \\
\text { for a COVID suspect or } \\
\text { confirmed case }\end{array}$ & Yes & Yes & Yes & - & $\begin{array}{l}\text { Yes, } \\
\text { full cover }\end{array}$ & - & $\begin{array}{r}\text { Yes, } \\
\text { and long } \\
\text { shoe cover }\end{array}$ \\
\hline Housekeeping staff & Yes & - & - & Yes & Yes & - & Yes \\
\hline Security officer & Yes & - & - & Yes & - & - & - \\
\hline Transport staff & Yes & - & - & Yes & - & Yes & Yes \\
\hline
\end{tabular}

HCP: healthacare provider; *goggles; HD: hemodialysis; PD: peritoneal dialysis.

- Access: Central venous catheter for HD or PD catheter should be placed with complete PPE. For patients who already have arteriovenous fistula, CRRT and SLED may be considered provided monitoring for potential complications of the procedure is possible [23].

- Prescribing CRRT: In centers having facility for CRRT, the treatment time for continuous veno-venous hemodialfiltration may be reduced to 10-12 hours in order to make the machine available for a greater number of patients. In case of shortage of replacement fluids, the dose could be reduced to $1000 \mathrm{ml} / \mathrm{m}^{2}$ instead of $2000 \mathrm{ml} / \mathrm{m}^{2}$, especially after first few-hour and once metabolic control is achieved. Normal saline may also be used as replacement fluid.

- Anticoagulation: Centers dialyzing patients with COVID-19 have reported circuit clotting in CRRT and SLED if anticoagulants are not used. Anticoagulation is done, as per unit protocol. In case of non-availability of pumps for heparin, low molecular weight heparin may be used (enoxaparin single dose $0.5-1 \mathrm{mg} / \mathrm{kg}$; dalteparin<15-kg: $1500 \mathrm{IU} ; 15-30 \mathrm{~kg}: 2500 \mathrm{IU}$ ) [24].

- In order to minimize exposure, the CRRT or HD machine may be set-up outside the patient area, and then taken into the room and connected.

- After treatment, all equipment should be disinfected with $1 \%$ sodium hypochlorite before being removed from the room.

- Transport of patients with suspected or confirmed COVID-19 to a central dialysis unit is not recommended. These patients should be dialyzed bedside, using portable reverse osmosis.

- Acute peritoneal dialysis should be considered when hemodialysis machines are not available. An automated cycler should be used to minimize patient contact. The drain fluid is disposed, are per protocol. All consumables like tubings, dialyzers and replacement solutions bags should be discarded.

\section{TRANSPLANT RECIPIENTS}

Kidney transplant recipients must be considered highly susceptible to SARS-CoV-2 infection. Data on COVID-19 in transplant patients is however limited. It has been observed that among adults renal transplant recipients with COVID-19, 60\% require hospitalization and $25-30 \%$ require ICU care with mortality rate of $5 \%[6,25]$. However, a recent study has shown high early mortality in transplant recipients than general population with Covid 19 infection [7].

\section{General Precautions}

Transplant recipients are advised to follow general precautions for patients with CKD.

- Movement outside the home, including for follow up hospital visits, should be restricted. Teleconsultation may be utilized to contact HCP.

- When outside the house, transplant recipients and caregivers should use triple-layer mask and prevent touching of nose and mouth.

- It is essential to maintain a 4-weeks stock of 
medications. If the family is unable to obtain medications, the transplant team should be informed.

\section{Transplantation During COVID-19 Pandemic}

Unlike other solid organs, kidney transplantation is performed in a relatively stable patient, receiving maintenance HD. Transplantation is associated with marked immunosuppression, which might not be in patient's interest during the pandemic. Transplant recipients may also require respiratory support and ICU monitoring during the peri-operative period, facilities that are scarce during the outbreak. Using these facilities for an elective procedure might also reduce their availability for a critically ill COVID-19 patient. We believe that the risks of performing kidney transplantation outweigh the benefits to either the patient or the healthcare system. We recommend postponing live-related donor transplants until the outbreak has abated.

The National Organ and Tissue Transplant Organization (NOTTO) has advised temporary suspension of deceased and live related transplant program [26]. However, if pandemic lasts for longer duration, then reconsideration of recommendation is advised.

\section{Transplant Recipients With COVID-19}

Transplant recipients presenting with cough or shortness of breath with or without fever, history of contact with known patient, or with features of SARI should be screened for SARS-CoV-2 infection by RT-PCR of nasopharyngeal swabs. These patients may have atypical features such as coryza, diarrhea and fatigue. Fever is reported in 50-87\%, while diarrhea and lymphopenia are observed in $30 \%$ and $50 \%$ patients, respectively $[6,23]$. One-third may have no radiographic findings. A high index of suspicion is necessary to diagnose COVID-19 in transplant recipients.

Supportive management for transplant recipients with COVID-19 is shown in Fig. 3. For patients with mild disease, reduction of immunosuppression is not recommended as this might result in allograft rejection. In sicker patients (with pneumonia, but not critically ill), the anti-proliferative agent (mycophenolate or azathioprine) should be discontinued. The dose of prednisolone is increased to $0.5-1 \mathrm{mg} / \mathrm{kg}$ when therapy with mycophenolate is stopped. Dose of calcineurin inhibitors (CNI) is reduced to target lower levels (Tacrolimus adjusted to achieve a trough of $4-6 \mathrm{ng} / \mathrm{mL}$; Cyclosporine $100-150 \mathrm{ng} /$ $\mathrm{mL}$ ). In critically ill children (requiring ventilation and inotropic support), CNI may be reduced further or discontinued $[27,28]$. Such patients are managed with steroid monotherapy, at a higher dose. Once recovery begins, immune-suppressants should be reintroduced and increased to pre-illness doses 14-days after two nasopharyngeal swabs are negative.

There is no evidence to support the use of antiviral treatment for COVID-19. Drugs being examined include lopinavir/ritonavir, remdesivir, favipiravir, HCQ, tocilizumab, interferon- $\alpha$ and intravenous immunoglobulins. Interaction of medications with $\mathrm{CNI}$ and sirolimus needs to be considered. Lopinavir/ritonavir, darunavir/ritonavir, chloroquine and HCQ can potentially increase CNI levels, while tocilizumab decreases CNI and sirolimus levels [29]. Other causes for fever, including bacterial or viral infections should be ruled out. Antibiotics should be used for empiric treatment of bacterial infections and modified based on culture sensitivity results.

\section{CONCLUSIONS}

The present guidelines of the Indian Society of Pediatric Nephrology on managing patients with kidney diseases during the COVID-19 pandemic are based on current literature and expert views. While children constitute a small proportion of patients with COVID-19, those with chronic disorders constitute a high-risk group and at-risk for adverse outcomes. Therapeutic guidelines are likely to change as evidence emerges from large case series and randomized controlled trials.

\section{REFERENCES}

1. Dong Y, Mo X, Hu Y, Qi X, Jiang F, Jiang Z, et al. Epidemiology of COVID-19 among children in China. Pediatrics. 2020 Mar 16. [Epub ahead of print]. Available from: https://pediatrics.aappublications.org/content/early/ 2020/03/16/peds.2020-0702.1. Accessed on May 14, 2020.

2. CDC COVID-19 Response Team. Coronavirus Disease 2019 in Children- United States, February 12-April 2, 2020. MMWR Morb Mortal Wkly Rep. 2020; 69:422-26.

3. Ministry of Health and Family Welfare, Government of India. COVID-19 INDIA. Available from: https:// www.mohfw.gov.in/. Accessed on May 8, 2020.

4. Ma Y, Diao B, Xifeng L, Zhu J, Liang W, Liu L, et al. 2019 novel coronavirus disease in hemodialysis patients: Report from one HD center in Wuhan, China. Available from: https://www.medrxiv.org/content/10.1101/2020.02.24. 20027201v2/. Accessed on April 12, 2020.

5. Alberici F, Delbarba E, Manenti C, Econimo L, Valerio F, Pola A, et al. A single center observational study of the clinical characteristics and short-term outcome of 20 kidney transplant patients admitted for SARS-CoV2 pneumonia. Kidney Int. 2020 Apr 9. [Epub ahead of print]. Available from: https://www.kidney-international. org/article/S00852538(20)30365-3/fulltext. Accessed on May 14, 2020

6. Columbia University Kidney Transplant Program. Early Description of Coronavirus 2019 Disease in Kidney Transplant Recipients in New York. J Am Soc 

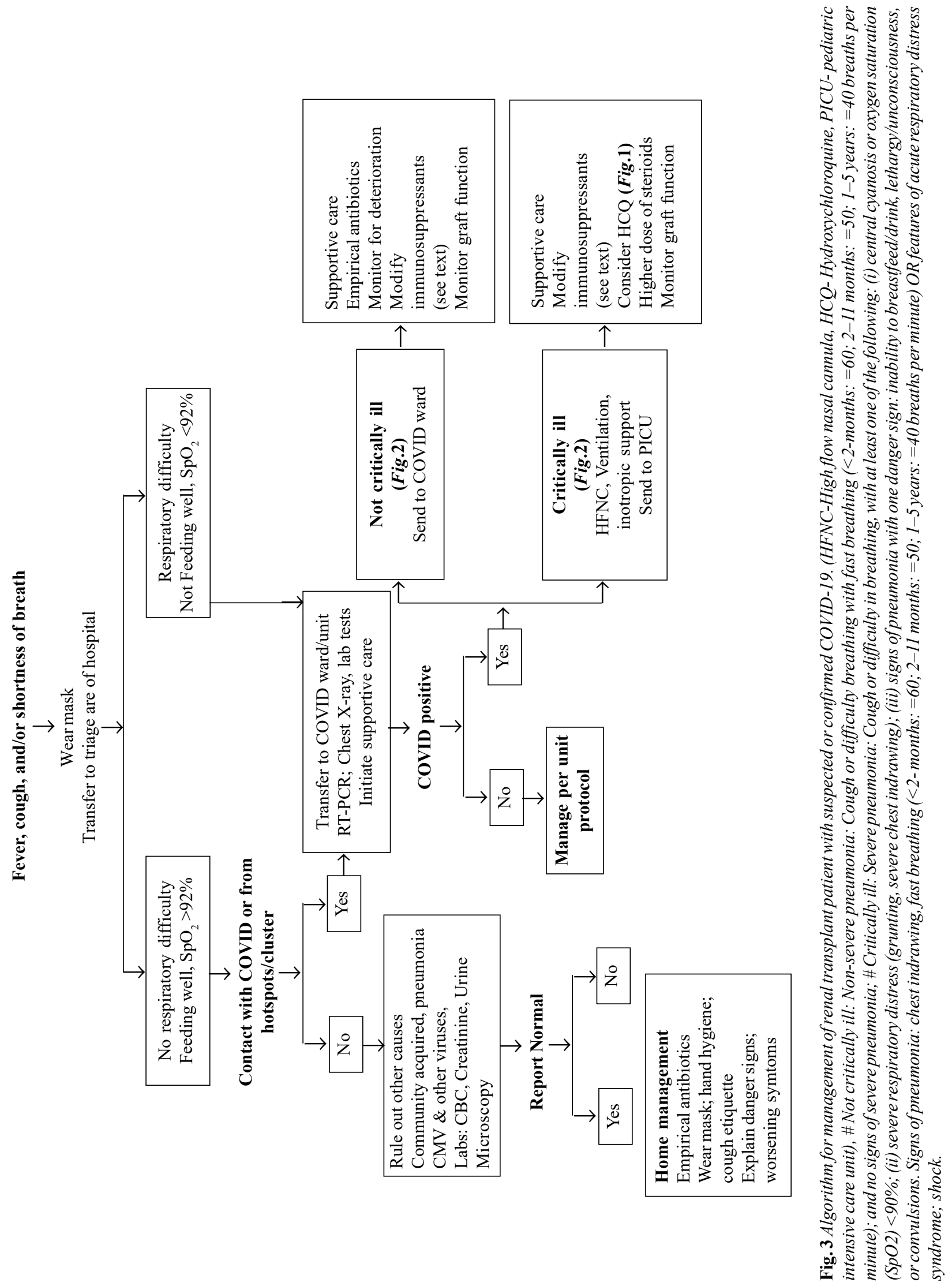
Nephrol. 2020 Apr 21. [Epub ahead of print]. Available from https://jasn.asnjournals.org/content/early/2020/04/ 27/ASN.2020030375.long. Accessed on May 14, 2020.

7. Akalin E, Azzi Y, Bartash R, Seethamraju H, Parides $\mathrm{M}$, Hemmige $\mathrm{V}$, et al. Covid 19 and kidney transplan-tation. $\mathrm{N}$ Engl J Med. 2020 Apr 24 [Epub ahead of print]. Available from: https://www.ncbi.nlm.nih.gov/pmc/articles/ PMC7200055/pdf/NEJMc2011117.pdf. Accessed on May 14, 2020.

8. Ministry of Health and Family Welfare Directorate General of Health Services (EMR division). Revised guidelines on clinical management of COVID-19. Available from: www.mohfw.gov.in/pdf/RevisedNationalClinical ManagementGuidelineforCOVID1931032020.pdfl. Accessed on April 26, 2020.

9. Board of Governors in Supersession of the Medical Council of India. Telemedicine Practice Guidelines. Available from: https://www.mohfw.gov.in/pdf/Telemedicine.pdfl. Accessed on April 26, 2020.

10. Ministry of Health and Family Welfare Directorate General of Health Services (EMR). Guidelines on rational use of personal protective equipment. Available from: www. mohfw.gov.in/pdf/Guidelinesonrationaluseof PersonalProtectiveEquipment.pdfl. Accessed on April 26, 2020.

11. Ministry of Health and Family Welfare Directorate General of Health Services (EMR). SOP for reallocation of residents/PG students and nursing students as part of hospital management of COVID. Available from: https:// www.mohfw.gov.in/pdf/COVID19SOPfordoctorsand nurses. pdfl. Accessed on April 26, 2020.

12. Indian Council of Medical Research. Recommendation for empiric use of hydroxychloroquine for prophylaxis of SARS-CoV-2 infection. Available from: https://icmr.nic.in/ sites/default/files/upload_documents/HCQ_Recommen dation_22March_final_MM_V2.pdfl. Accessed on April 26, 2020.

13. Wallace $\mathrm{D}$, on behalf of BAPN. Information and guidance for children on hemodialysis, peritoneal dialysis and immune suppression (including renal transplants). Available from: https://renal.org/covid-19/paediatricuseful-information-resources/. Accessed on April 26, 2020.

14. For patients with CKD using immunosuppressive therapy. Available from: https://renal.org/stratified-risk-prolongedself-isolation-adults-children-receiving-immuno suppression-disease-native-kidneys/. Accessed on April 25, 2020.

15. Liu Z, Xiao X, Wei X, Li J, Yang J, Tan H, et al. Composition and divergence of coronavirus spike proteins and host ACE2 receptors predict potential intermediate hosts of SARSCoV-2. J Med Virol. 2020 Feb 26. [Epub ahead of print]. Available from: https://onlinelibrary. wiley.com/doi/full/10.1002/jmv.25726. Accessed on May 14,2020

16. Vaduganathan M, Vardeny O, Michel T, McMurray JJV, Pfeffer MA, Solomon SD. Renin-angiotensin-aldosterone system inhibitors in patients with Covid-19. N Engl J Med. 2020;382:1653-59.
17. Covid-19 clinical trials tracker. Available from: https:// covidclinicaltrials.com. Accessed on April 26, 2020.

18. Indian Council of Medical Research. Testing Labs Details. Available from: https://icmr.nic.in/sites/default/files/ upload_documents/Strategey_for_COVID19_Test_v4_ 09042020.pdf/. Accessed on April 07, 2020.

19. Ministry of Health and Family Welfare. Revised Guidelines for Dialysis of COVID - 19 patients. Available from: https://www.mohfw.gov.in/pdf/RevisedGuidelinesfor DialysisofCOVID19Patients.pdf. Accessed on April 26, 2020.

20. Schwierzeck V, König JC, Kühn J, Mellmann A, CorreaMartínez CL, Omran H, et al. First reported nosocomial outbreak of severe acute respiratory syndrome coronavirus 2 (SARS-CoV-2) in a pediatric dialysis unit. Clin Infect Dis. 2020 Apr 27. [Epub ahead of print]. Available from: https:/ lacademic.oup.com/cid/advance-article/doi/10.1093/cid/ ciaa491/5825509. Accessed on May 14, 2020.

21. Shen Q, Wang M, Che R, Li Q, Zhou J, Wang F, et al; Chinese Society of Pediatric Nephrology and Chinese Medical Doctor Association of Pediatric Nephrology. Consensus recommendations for the care of children receiving chronic dialysis in association with the COVID-19 epidemic. Pediatr Nephrol. 2020 Apr 24. [Epub ahead of print]. Available from: https://link.springer.com/article/ 10.1007/s00467-020-04555-x. Accessed on May 14, 2020.

22. Naicker S, Yang CW, Hwang SJ, Liu BC, Chen JH, Jha V. The novel Coronavirus 2019 epidemic and kidneys. Kidney Int. 2020; 97: 824-28.

23. Al Rifai A, Sukul N, Wonnacott R, Heung M. Safety of arteriovenous fistulae and grafts for continuous renal replacement therapy: Michigan experience. Hemodial Int. 2018;22:50-55.

24. Davenport A. Alternatives to standard unfractionated heparin for pediatric hemodialysis treatments. Pediatr Nephrol 2012; 27:1869-79.

25. Hiremath S, Topf JM. Transplant. Available from: www.nephjc.com/news/covidtx/. Accessed on April 07, 2020.

26. National transplant specific guidance for COVID-19. Available from: https://notto.gov.in/WriteReadData/Portal/ News/711_1_FINAL_GUIDANCE_COVID-19_ 31.03.2020.p. $d \bar{f} /$. Accessed on April 22, $20 \overline{2} 0$.

27. Guidance on the management of transplant recipients diagnosed with or suspected of having COVID19. Available from: https://bts.org.uk/wpcontent/uploads/2020/03/ Clinical_management_transplant_recipients.pdf/. Accessed on April 07, 2020.

28. DESCARTES expert opinion regarding the management of immunosuppressive medication for kidney transplant patients during the COVID-19 pandemic. Available from: https://www.era-edta.org/en/wp-content/uploads/2020/04/ Expert-opinion-on-ISD-in-Covid-19.pdf /. Accessed on April 07, 2020.

29. Characteristics of potential antiviral agents under evaluation for treatment of COVID-19. Available from: https:// www.covid19treatmentguidelines.nih.gov/thera peuticoptions-under-investigation/. Accessed on April 26, 2020. 